\title{
Thermal diagnostic of the optical window on board LISA Pathfinder
}

\author{
M Nofrarias ${ }^{1}$, A F García Marín ${ }^{2}$, A Lobo ${ }^{1,3}$, G Heinzel $^{2}$, \\ J Ramos-Castro ${ }^{4}$, J Sanjuán ${ }^{1}$ and K Danzmann ${ }^{2}$ \\ ${ }^{1}$ Institut d'Estudis Espacials de Catalunya (IEEC), Edifici Nexus, Gran Capità 2-4, \\ 08034 Barcelona, Spain \\ 2 Max-Planck-Institut für Gravitationsphysik (Albert-Einstein-Institut), Callinstrasse 38, \\ D-30167 Hannover, Germany \\ ${ }^{3}$ Institut de Ciències de 1'Espai, CSIC, Facultat de Ciències, Torre C5 parell, 08193 Bellaterra, \\ Spain \\ ${ }^{4}$ Departament d'Enginyeria Electrònica, UPC, Campus Nord, Edifici C4, Jordi Girona 1-3, \\ 08034 Barcelona, Spain \\ E-mail: nofraria@ieec.uab.es
}

Received 11 June 2007, in final form 21 August 2007

Published 2 October 2007

Online at stacks.iop.org/CQG/24/5103

\begin{abstract}
Vacuum conditions inside the LTP gravitational reference sensor must be under $10^{-5} \mathrm{~Pa}$, a rather demanding requirement. The optical window $(\mathrm{OW})$ is an interface which seals the vacuum enclosure and, at the same time, lets the laser beam go through for interferometric metrology with the test masses. The OW is a plane-parallel plate clamped in a titanium flange, and is considerably sensitive to thermal and stress fluctuations. It is critical for the required precision measurements, hence its temperature will be carefully monitored in flight. This paper reports on the results of a series of OW characterization laboratory runs, intended to study its response to selected thermal signals, as well as their fit to numerical models, and the meaning of the latter. We find that a single-pole ARMA transfer function provides a consistent approximation to the OW response to thermal excitations, and derive a relationship with the physical processes taking place in the OW. We also show how the system noise reduction can be accomplished by means of that transfer function.
\end{abstract}

PACS numbers: $04.80 . \mathrm{Nn}$, 95.55.Ym, 04.30.Nk, 07.87.+v, 07.60.Ly, 42.60.Mi

(Some figures in this article are in colour only in the electronic version)

\section{Introduction}

LISA Pathfinder (LPF) is an ESA mission, with NASA contributions, whose main objective is to put to test critical parts of the Laser Interferometer Space Antenna (LISA), the first spaceborne gravitational wave $(\mathrm{GW})$ observatory [1]. The science module on board $L P F$ is the 
LISA Technology Package (LTP) [2]. The unprecedented sensitivity of the LTP has prompted the conceptual enhancement of $L P F$ 's science objectives as regards the purity of geodesic, or free-fall motion of test masses in the interplanetary gravitational field $[3,5]$.

Free fall control is achieved by gravitational reference sensors (GRS) [6]. These are a set of capacitive sensors which can determine to high precision (nanometres), the 3D position and orientation of cubic test masses relative to their non-contacting enclosure, which is rigidly linked to the spacecraft structure. Detected off-centre deviations trigger action by a set of micro-thrusters which move the spacecraft such that the mass returns to its centred position. The combination of the GRS, the thrusters and the control system (the drag-free and attitude control system (DFACS)) is called the drag-free subsystem. The latter is intended to accurately nullify the effects of any non-gravitational forces acting on the spacecraft. This makes it possible to detect differential gravitational accelerations between the two test masses, whether by precision interferometry [7] or by the drag-free system itself.

This is fundamental for LISA, since gravitational waves (GWs) show up as tides, i.e., time-varying differential gravitational accelerations. The precision of the measurement done with the LTP is required to be [8]

$S_{\Delta a}^{1 / 2}(\omega) \leqslant 3 \times 10^{-14}\left[1+\left(\frac{\omega / 2 \pi}{3 \mathrm{mHz}}\right)^{2}\right] \mathrm{m} \mathrm{s}^{-2} \mathrm{~Hz}^{-1 / 2}, \quad 1 \mathrm{mHz} \leqslant \frac{\omega}{2 \pi} \leqslant 30 \mathrm{mHz}$.

We shall refer to the above frequency band as the LTP measuring bandwidth (MBW) in the following. Equation (1) is ten times less demanding than what is needed for LISA [9], both in magnitude and in frequency band, yet it is between two and three orders of magnitude better than has been achieved or required so far for space missions [3]. It has relevant consequences for future missions, which need high performance drag free, hence the relevance of $L P F$ beyond its natural objectives as a LISA precursor.

In order to meet the above requirements, the residual pressure inside the GRS must be under $10^{-5} \mathrm{~Pa}$, a condition which is classified as very high vacuum by the American Vacuum Society. This implies that the interior has to be tightly sealed within a vacuum enclosure (VE), and non-mechanical getter pumps installed to ensure a suitably rarefied environment around the test masses. Perhaps a more obvious option would have been to communicate the VE directly with the external interplanetary vacuum, which is much better than $10^{-5} \mathrm{~Pa}$. Recent studies [4] by the Project Engineering Team have shown that there are serious difficulties with such an option. For example, venting out of residual gas has time scales exceeding the very $L P F$ mission lifetime, cleanliness control inside the VE is tighter with the $\mathrm{OW}$, etc.

The general layout is shown in figure 1. As regards the issues we address in this paper, attention is drawn to the optical window (OW), which is the interface between the test masses and the Optical Bench: laser beams must bounce off the test masses to monitor their positions by precision interferometry, hence a transparent window is necessary for the light to make it to the interior of the VE.

The OW is a plane-parallel plate and is therefore a potential source of noise: random variations of its optical properties may result in corresponding optical path fluctuations, which distort the laser light phase, hence the optical metrology readout. Great care must be taken when manufacturing this critical component of the LTP and, once manufactured, characterization of its behaviour duly performed.

The most important agent responsible for OW fluctuations is temperature fluctuations. These cause various degrees of mechanical stresses across the rim, as well as temperature dependent index of refraction changes. The former are very difficult to model with quantitative accuracy, mostly due to lack of precise control of mounting interface behaviour, but the former can be much better studied in a stress free environment. This paper is concerned with the 


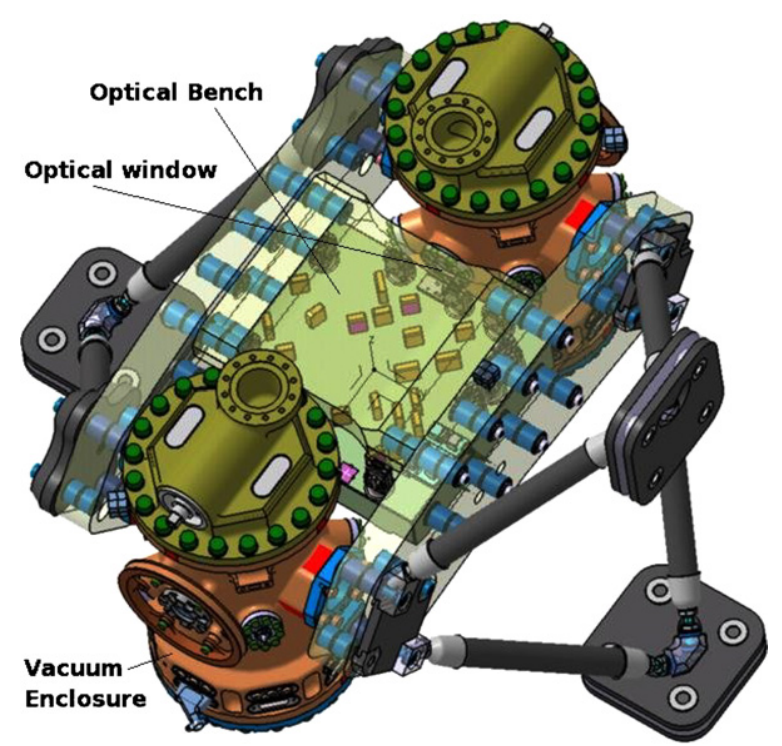

Figure 1. Layout of the LTP core assembly, as of 13 October 2006. The test masses are Au-Pt alloy cubes inside either vacuum enclosure.

experimental characterization on ground of prototype OWs, and with the phenomenological modelling of their response to thermal excitations. This is justified if the noise fluctuations are smaller than the applied stimuli and if the system behaves linearly.

The philosophy of the approach is the one typical of the diagnostics subsystem, as described in [10]. This is: apply controlled temperature signals of high signal-to-noise ratio to the titanium flange where the OW is held (see below), and measure the temperature of the former. Measure also the induced phase shifts in a laser beam that travels through the OW, then try to establish the transfer function between both magnitudes, i.e., temperature and phase shifts. The transfer function thus obtained is also assumed to be valid in the situation when only noise is present in the flange. The latter extrapolation hypothesis is the clue to the determination of the phase noise contributed by the temperature fluctuation noise in the OW, on the basis of temperature measurements.

The success of the proposed empirical approach depends on our ability to find a transfer function which depends on a (preferably reduced) number of parameters, which does not change significantly across different conditions and runs of the experiment. As we shall now show, we have found that a single-pole $A R M A^{5}$ process describes rather satisfactorily the relationship we look for. The precise meaning of this concept will be discussed in detail in the following sections, and the results applied to evaluate the temperature fluctuation noise in a dedicated experiment.

The paper is organized as follows. In section 2 we describe the experiment layout, including hardware and data acquisition details. Section 3 is devoted to the data processing, analysis, two modes of model fitting - a direct linear regression and a single-pole ARMA model - and numerical results. In section 4, we examine in detail the $\operatorname{ARMA}(2,1)$ fit, and derive important implications for the understanding of the physical processes happening in the OW. Section 5 addresses how the previous analysis can be applied to quantify the

\footnotetext{
5 Acronym for auto regressive moving average, roughly the discrete time series equivalent of a linear differential
} equation (see a standard textbook, e.g., [14]). 

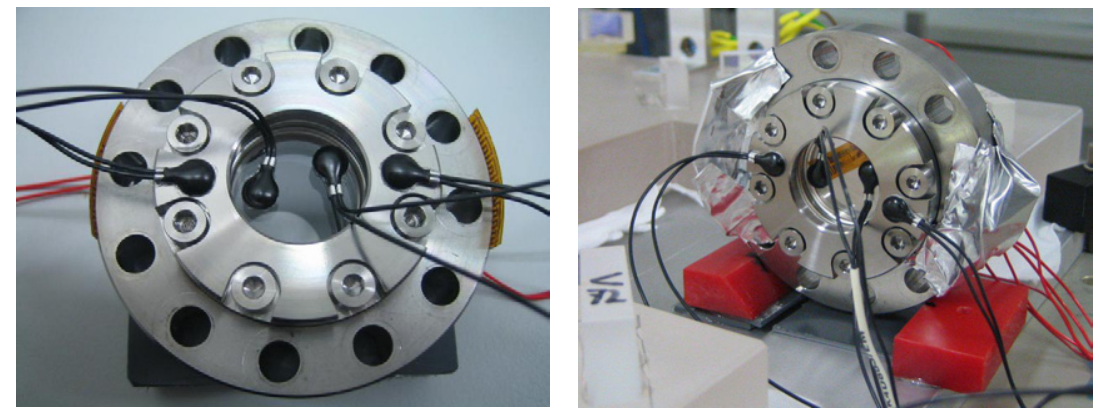

Figure 2. The OW (left), with the plane-parallel plate inside the titanium flange, heaters on the lateral surface of the latter (pale brown foils) and two pairs of NTC temperature sensors (black beads with wires). On the right, mounting of the $\mathrm{OW}$ on rails for measurements. Note heaters are covered with aluminium foils: this is to prevent undesired heating of other components by the heaters' emission of thermal radiation.

contribution of temperature fluctuation noise to the total phasemeter noise, based on another set of experimental results, and section 6 develops an interesting exercise whereby a continuous time model is suggested as the origin of the discrete time ARMA fit. Finally, conclusions and bibliographic references close the article.

\section{Experiment description}

The current baseline of LISA Pathfinder and LISA includes vacuum tanks containing the test masses which act as end mirrors for the interferometer. The presence of such tanks, or vacuum enclosures (VE), forces the inclusion of a transmissive element interfacing between the interior of the VE and the optical bench outside. This optical element is the optical window (OW). In this section, we describe the laboratory hardware and conditions of several runs of measurements conducted in AEI Hannover Laboratory facilities to characterize the thermal behaviour of the OW.

In the experiment two different prototype OWs were tested. Both were manufactured following the same baseline as the one to be applied in the final LTP flight model. The main element of the window is a very low thermal expansion coefficient glass chosen in order to minimize the variation of the optical pathlength with respect to the temperature. The figure of merit-quantified by equation (21) - is $0.59 \times 10^{-6} \mathrm{~K}^{-1}$ for our particular choice, the OHARA S-PHM52 $\left(n=1.606, \mathrm{~d} n / \mathrm{d} T=-5.54 \times 10^{-6} \mathrm{~K}^{-1}\right)$. This parameter can reach values as high as $5.31 \times 10^{-6} \mathrm{~K}^{-1}$ for BK7 or $8.32 \times 10^{-6} \mathrm{~K}^{-1}$ for fused silica. The glass of $30 \mathrm{~mm}$ diameter and $6 \mathrm{~mm}$ length was clamped between two titanium flanges, fastened by means of titanium bolts and sealed by two helicoflex rings ${ }^{6}$ to prevent gas leakage in space conditions.

The OW is expected to induce thermal-related noise in the metrology subsystem. In order to quantify its contribution to the total noise budget a set of thermal diagnostics items were attached to the optical window prototypes. They are shown in figure 2, left panel: two Kapton heaters Minco HK5303 attached to the titanium flange lateral surface, and four glass encapsulated thermistors Betatherm G10K4D853 attached in pairs to the titanium flange and on the athermal glass surface, for precision temperature measurements. These diagnostics items were all glued to their attachment points with pressure sensitive adhesive (PSA) tape $3 M-966$ of similar characteristics to the one to be used in flight. The temperature sensors on

\footnotetext{
6 These are softer metallic rings, e.g. aluminium or silver.
} 


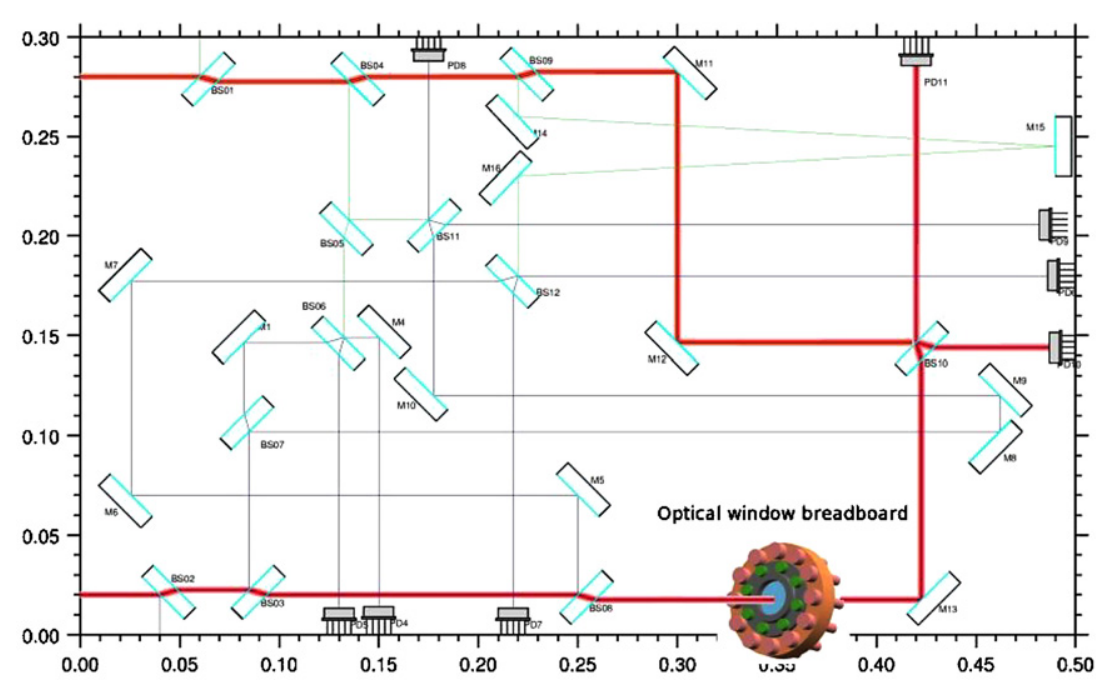

Figure 3. Schematic of the interferometric measurement concept.

the glass will actually not fly with the LTP. They will however provide relevant information to implement real mission data analysis procedures and methods, for which only the titanium temperature data will be available.

During the experiment, the window was leaning vertically on a PVC two-rail structure (see figure 2, right panel), which impeded any high conductivity thermal contact with the rest of the hardware. Although not directly affecting the thermo-optical interaction studied here, the OW will be part of the VE in the real LTP, thus a higher thermal conductance is to be expected, and therefore a faster suppression of thermal gradients is foreseen during mission operations.

The complete setup (i.e., the glass plus its mounting structure and the just-mentioned diagnostics items) was inserted as a transmissive element in a dedicated optical bench, as seen in figure 3. The heaters were covered with aluminium foil to reduce thermal radiation effects (figure 2, right). For the same reason, the window was introduced in a copper box leaving only a narrow opening for the laser beam to go through. As seen in the schematic of figure 3 , the beam traverses the OW only once. This will not be the case in the real LTP, where the laser will go twice through each window, instead, but the one passage configuration used here simplifies the OW thermal characterization without information losses. All the experiments were performed under low-pressure conditions at a $P \simeq 10^{-3} \mathrm{~Pa}$ vacuum level.

The optical window was subjected to various heat pulses comprising a wide range of duration and powers in order to identify suitable parameters for the thermal test to be performed in-flight. The data here reported gather 25 experiment runs on two different prototypes, applying heat pulses from $100 \mathrm{~mW}$ to $2 \mathrm{~W}$ ranging from $10 \mathrm{~s}$ to $100 \mathrm{~s}$ of application time. All experiments were performed at room temperature, which falls within the expected range of working temperatures of the $L T P$ experiment during operations, required to be between $10^{\circ} \mathrm{C}$ and $30^{\circ} \mathrm{C}$. Figure 4 shows a typical response data plot, with indication of the temperature sensor readings and the interferometrically registered phase shifts corresponding to a specific heat signal input (see the figure caption for the details).

Two different data acquisition systems were used in the experiment: the interferometric data were acquired via the LTP phasemeter prototype [7], whereas the thermal diagnostics data 


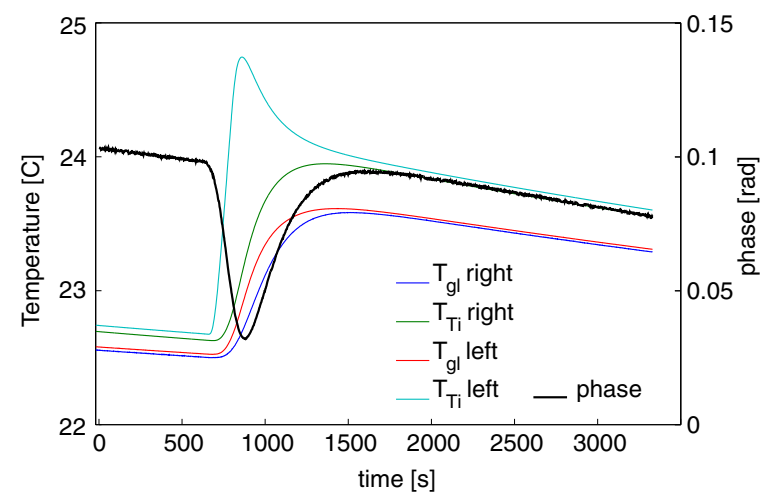

Figure 4. Phase (black curve) and various temperature sensors' responses (coloured curves) to a $2 \mathrm{~W}$ heat pulse applied for $100 \mathrm{~s}$. Legend indications correspond to the thermometers shown in figure 2 .

were acquired using the LTP front end electronics (FEE) prototype [11, 12]. Both acquisition systems have previously successfully passed tests of compliance with mission noise budgets.

\section{Fitting the data to models}

The main purpose of this section is to give an account of the measured interferometer output data in terms of the also measured temperature data. While in this experiment both are of course ultimately caused by the heaters' signal, our interest focuses on the temperature versus phase relationship, as this is the one we need to quantify the magnitude of temperature fluctuations noise during science operations in flight [10].

To serve this purpose, we adopt model-fitting techniques. Two approaches will be proposed, and discussed in the ensuing section: a direct linear regression fit of the interferometric data to the temperature read-out coming from sensors on the titanium flange and those on the OW glass itself, and an ARMA model using only temperature readout from the titanium temperature sensors. The latter is of particular interest, since it is not foreseen that temperature sensors will be attached to the glass surface in the real LTP.

\subsection{Data conditioning}

Before we attempt to fit the data to a useful model, some data pre-processing is required.

The temperature and phase acquisition data systems reside on different hardware and software, and deliver the respective time series data for analysis at sample rates which are different as well: temperature data are sampled at $f_{\mathrm{s}, T}=0.65 \mathrm{~Hz}$, whereas phase data are sampled at $f_{\mathrm{s}, \phi}=32.4 \mathrm{~Hz}$, instead. Downsampling and resampling thus needs to be applied to the latter in order to make meaningful sense of data fitting algorithms. To avoid aliasing effects at downsampling phase, suitable low-pass filters are applied. This is however not enough to have matched sampling times in both time series, so an additional interpolation algorithm is used for properly matched resampling.

In addition, each data segment is de-trended prior to model fitting. The removed trend is evaluated from the first $500 \mathrm{~s}$ previous to the heat input signal beginning. This way we get rid of environmental drifts and spurious trending effects. 


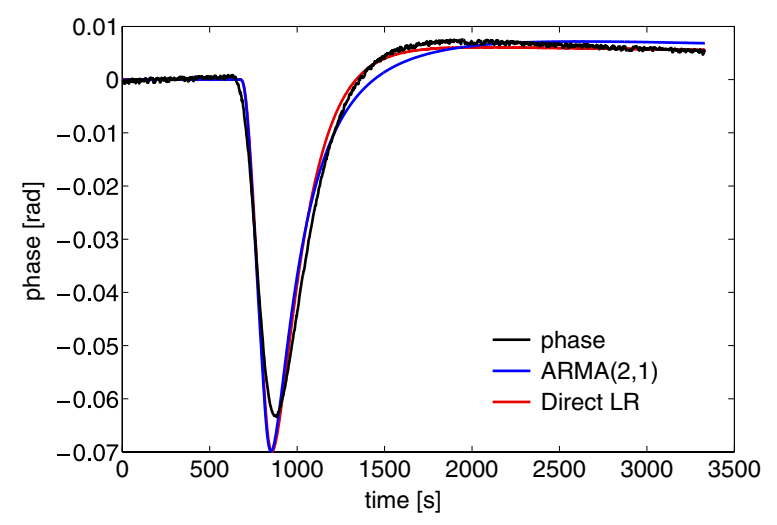

Figure 5. Example of fit results for two different models.

\subsection{Direct linear regression}

A typical phasemeter response when heat pulses are applied to the OW is shown in figure 4. An essentially instantaneous phase response is observed in coincidence with thermometers' excitations, which suggests phase behaviour can be described as a direct, or single-time relationship between the various temperature readings and associated phase shifts. If we additionally make the hypothesis that such a relationship is linear ${ }^{7}$, then the model is given by

$$
\phi(t)=p_{1} T_{\mathrm{Ti}}(t)+p_{2} T_{\mathrm{Glass}}(t),
$$

where $T_{\mathrm{Ti}}(t)$ is the temperature read by the thermometer on the titanium flange closest to the activated heater, and $T_{\mathrm{Glass}}(t)$ that of a thermometer on the OW glass. The parameters $p_{1}$ and $p_{2}$ are to be estimated by a least-squares algorithm, which requires the square error

$$
\epsilon^{2}=\sum_{n=1}^{N}\left\{\phi[n]-p_{1} T_{\mathrm{Ti}}[n]-p_{2} T_{\mathrm{Glass}}[n]\right\}^{2}
$$

to be the smallest possible for the given data streams. Here, $\phi[n]$ is the $n$th sample of the measured phase, and $T_{\mathrm{Ti}}[n]$ and $T_{\mathrm{Glass}}[n]$ the corresponding temperature samples. Thus, for example,

$$
\phi[n] \equiv \phi(n \Delta t), \quad T_{\mathrm{Ti}}[n] \equiv T_{\mathrm{Ti}}(n \Delta t),
$$

etc, where the sampling time $\Delta t$ has been set to $\Delta t \equiv 1 / f_{\mathrm{s}, \mathrm{Temp}}$, as discussed in section 3.1. Finally, $N$ is the number of analysed samples of each read-out.

The conditions of minimum square error are of course given by the two equations

$$
\frac{\partial \epsilon^{2}}{\partial p_{1}}=\frac{\partial \epsilon^{2}}{\partial p_{2}}=0
$$

which, once solved, give least-squares estimates $\widehat{p}_{1}$ and $\widehat{p}_{2}$ of the parameters $p_{1}$ and $p_{2}$, respectively. An example of this procedure is shown in figure 5. We report on the results of this analysis in section 3.4.

7 This is, in fact, quite accurate, on account of the rather small temperature and phase variation ranges detected in the experiment. 


\subsection{ARMA model fit}

Although the linear regression method performs quite acceptably well, there is a clear motivation to find a model able to fit the data independently of the glass temperature readings, since the latter will not be available in flight.

In this section, we take a different approach to fit phase data to titanium only temperature readings. We shall now assume that the relationship between these magnitudes can be expressed by a dynamical equation. More specifically, we make the hypothesis that the phase is the output of a linear ARMA process $^{8}$, whose input is the temperature of the titanium, as recorded by the thermometer next to the activated heater. We express this by the formula [15]

$$
\phi[n]=G(q, \boldsymbol{\theta}) T_{\mathrm{Ti}}[n],
$$

where $G(q, \theta)$ is a rational expression of the type

$$
G(q, \boldsymbol{\theta})=\frac{\alpha_{0}+\alpha_{1} q^{-1}+\cdots+\alpha_{r} q^{-r}}{1+\beta_{1} q^{-1}+\cdots \beta_{s} q^{-s}}
$$

with $q$ representing the shift operator:

$$
q x[n]=x[n+1], \quad q^{-1} x[n]=x[n-1]
$$

for any discrete series $x[n]$. Finally, $\boldsymbol{\theta}$ is an abbreviation for the vector of $r+s+1 A R M A$ parameters $\alpha_{0}, \ldots, \alpha_{r}, \beta_{1}, \ldots, \beta_{s}$.

System identification in this approach is again based on a least-squares criterion, for which a suitably defined square error needs to be defined. Following [15], this is the so-called prediction error:

$$
\epsilon^{2}(\boldsymbol{\theta})=\sum_{n=1}^{N}\left\{\phi[n]-G(q, \boldsymbol{\theta}) T_{\mathrm{Ti}}[n]\right\}^{2} .
$$

The estimates $\widehat{\boldsymbol{\theta}}$ of the parameters $\boldsymbol{\theta}$ are those which cause $\epsilon^{2}(\boldsymbol{\theta})$ to be minimum. Algorithms to find them are more robust if the additional hypothesis holds that the residuals $\left\{\phi[n]-G(q, \boldsymbol{\theta}) T_{\mathrm{Ti}}[n]\right\}$, where $\phi[n]$ and $T_{\mathrm{Ti}}[n]$ are the actually recorded data, are a white noise sequence [15]. Reassuringly, this is quite accurately true for our data. An example result of the fit is shown in figure 5, blue curve (this figure is in colour only in the electronic version).

\subsection{Numerical results}

As stated in section 2, up to 25 rounds of measurements were carried through during the experiment. This is a considerable number which enables us to check the consistency of the fitting models just described. The methodology we have adopted is the following: for each run, we de-trend the data and then fit them to both the direct linear regression (DLR) and the ARMA models. Parameter estimates are then filed for further analysis, as we now describe. An observation on the ARMA fit is however in order before we proceed.

Indeed, in the ARMA fit we also need to make a choice of order of the process, i.e., we need to set the number of $\alpha \mathrm{s}$ and $\beta \mathrm{s}$ in equation (7). It turns out that an $\operatorname{ARMA}(2,1)$, i.e., two $\alpha$ s and one $\beta$, is an excellent approach, in the sense that differences between model predictions and actual phase data are kept small to a rather satisfactory level. Finer tuning can be accomplished adding more $\alpha \mathrm{s}$ and/or $\beta \mathrm{s}$, but only at the expense of excessive parameter estimates' dispersion across different runs. This is highly undesirable and hence we confine our model to the $A R M A(2,1)$.

\footnotetext{
8 We feel again justified in assuming a linear relationship by the small variation intervals of the magnitudes involved.
} 
Table 1. Mean values and rms variances of parameter estimates.

\begin{tabular}{ll}
\hline DLR & $\operatorname{ARMA}(2,1)$ \\
\hline$p_{1}=(-38 \pm 4) \times 10^{-3} \mathrm{rad} \mathrm{K}^{-1}$ & $\alpha_{0}=(39.6 \pm 3) \times 10^{-3} \mathrm{rad} \mathrm{K}^{-1}$ \\
$p_{2}=(65 \pm 20) \times 10^{-3} \mathrm{rad} \mathrm{K}^{-1}$ & $\alpha_{1}=(-39.5 \pm 3) \times 10^{-3} \mathrm{rad} \mathrm{K}^{-1}$ \\
& $\beta_{1}=-0.996 \pm 0.001$ \\
\hline
\end{tabular}

Table 1 summarizes the results of the analysis, and figure 6 shows the binned distribution of the parameter estimates across the 25 runs. An outstanding characteristic of the fit is that the two $M A$ coefficients very accurately verify the numerical relationship $\alpha_{1}=-\alpha_{0}$. The model thus relates the output phase data to the time derivative of the titanium temperature-we come back to this in section 4 .

There are a few important aspects of these results which are worth stressing:

- $\alpha_{1} \simeq-\alpha_{0}$, although the difference between their values is much less than their variances.

- Well within tolerance, $-\alpha_{1}=\alpha_{0}=p_{1}$.

- $\beta_{1}$ is strongly peaked at a nominal value, with only $0.1 \%$ relative tolerance.

- $p_{2}$ is much more disperse, with almost $30 \%$ variability.

\section{The $\operatorname{ARMA}(2,1)$ transfer function}

In view of the above remarks, it is expedient to rewrite the $A R M A(2,1)$ model in terms of the following parameters:

$$
\alpha \equiv-\alpha_{1}, \quad \delta \equiv \alpha_{0}+\alpha_{1}, \quad \beta \equiv \beta_{1} .
$$

Hence,

$$
G(z, \alpha, \beta, \delta)=\alpha \frac{1-z^{-1}}{1+\beta z^{-1}}+\frac{\delta}{1+\beta z^{-1}}
$$

is the $z$-transform of the process transfer function-we have replaced the shift operator $q$ by the complex variable $z$ [14]. It is also expedient to emphasize the structure of this formula as follows:

$$
G(z, \alpha, \beta, \delta)=\alpha G_{\mathrm{HP}}(z, \beta)+\delta G_{\mathrm{LP}}(z, \beta)
$$

with

$$
G_{\mathrm{HP}}(z, \beta) \equiv \frac{1-z^{-1}}{1+\beta z^{-1}}, \quad G_{\mathrm{LP}}(z, \beta) \equiv \frac{1}{1+\beta z^{-1}}
$$

We now find discrete Fourier transforms (DFT) by the substitution

$$
z=\exp (\mathrm{i} \omega \Delta t)
$$

where $\Delta t$ is the sampling time of the time series data. The following obtains:

$$
\begin{aligned}
& \left|\widetilde{G}_{\mathrm{HP}}(\omega, \beta)\right|^{2}=\frac{4 \sin ^{2}(\omega \Delta t / 2)}{1+2 \beta \cos (\omega \Delta t)+\beta^{2}} \\
& \left|\widetilde{G}_{\mathrm{LP}}(\omega, \beta)\right|^{2}=\frac{1}{1+2 \beta \cos (\omega \Delta t)+\beta^{2}} \\
& \mid \widetilde{G}_{\left.(\omega, \alpha, \beta, \delta)\right|^{2}}=\frac{\delta^{2}+4 \alpha(\alpha+\delta) \sin ^{2}(\omega \Delta t / 2)}{1+2 \beta \cos (\omega \Delta t)+\beta^{2}} .
\end{aligned}
$$


We thus see that the transfer function $G$ is the sum of a high-pass term, $G_{\mathrm{HP}}$, and a low-pass term, $G_{\mathrm{LP}}$. The effect of the latter is naturally dominant at low frequencies, while the high-pass term dominates at high frequencies. The concepts of low and high frequencies can be made precise by means of some intermediate frequency $f_{\mathrm{b}}$ where the gains of $G_{\mathrm{HP}}$ and $G_{\mathrm{LP}}$ are equal. This is easily calculated:

$$
f_{\mathrm{b}} \simeq\left|\frac{\delta}{\alpha}\right| \frac{1}{2 \pi \Delta t}
$$

and has a numerical value of $f_{\mathrm{b}} \simeq 0.2 \mathrm{mHz}$, which means the high-pass effect dominates throughout the LTP bandwidth. We may not however neglect the relevance of the low pass at lower frequencies, as it contributes extremely valuable information for LISA.

A Bode diagram representation for the transfer functions is shown in figure 7 . The filter modulus is characterized by a plateau of $|\widetilde{G}| \sim 40 \times 10^{-3} \mathrm{rad} \mathrm{K}^{-1}$ across the entire LTP measuring bandwidth. Temperature fluctuations at frequencies below this bandwidth are also suppressed but following different behaviour, related to the low-frequency response of the optical window. The figure also shows the phase behaviour of the filter, and indicates increasing delays for high-frequency perturbations.

The DC gain of the filter is

$$
|\widetilde{G}(\omega=0, \alpha, \beta, \delta)|=\frac{\delta}{1+\beta} .
$$

If the estimated parameters are substituted into this expression then the following is obtained:

$$
|\widetilde{G}(\omega=0, \alpha, \beta, \delta)|=(25 \pm 4) \times 10^{-3} \mathrm{rad} \mathrm{K}^{-1}
$$
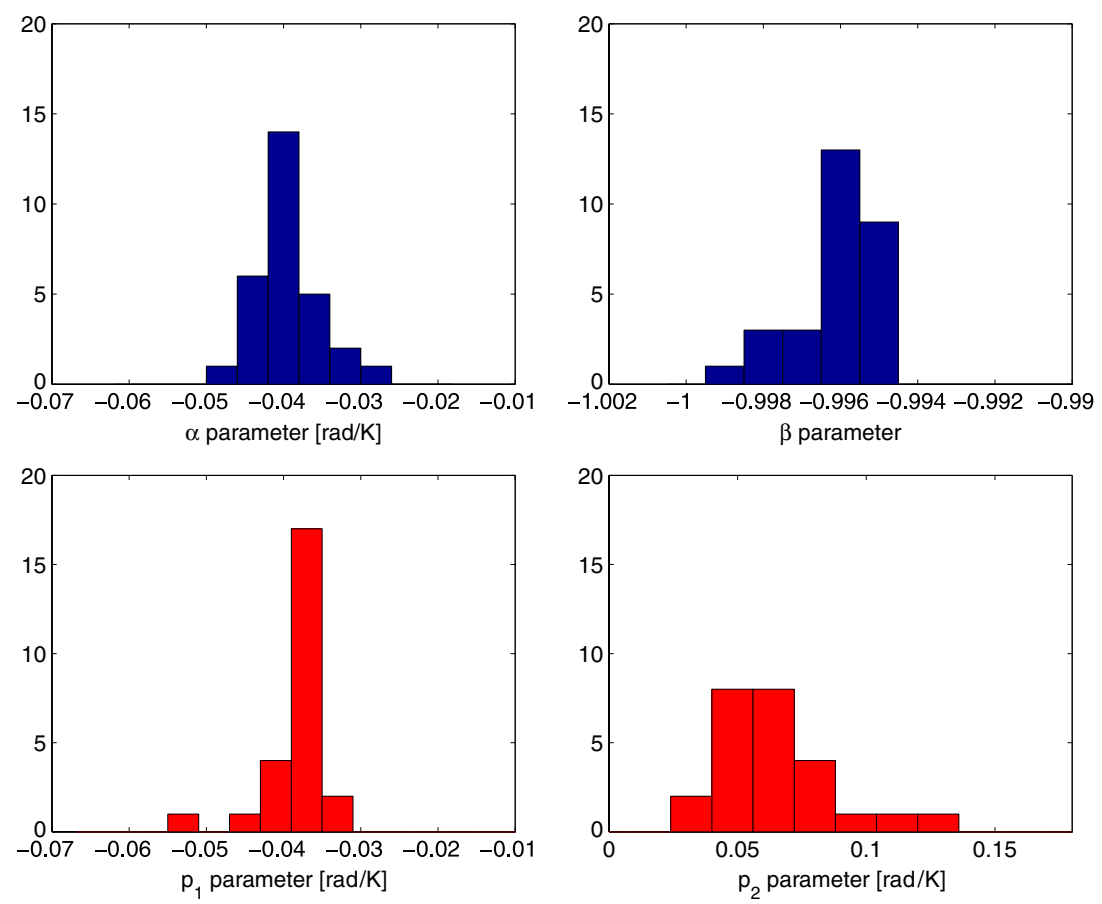

Figure 6. Histograms of the estimated parameters for the two fitting models described in the text. Top: $\operatorname{ARMA}(2,1)$, with $\alpha \equiv \alpha_{0}$ and $\beta \equiv \beta_{1}$. Bottom: DLR. 

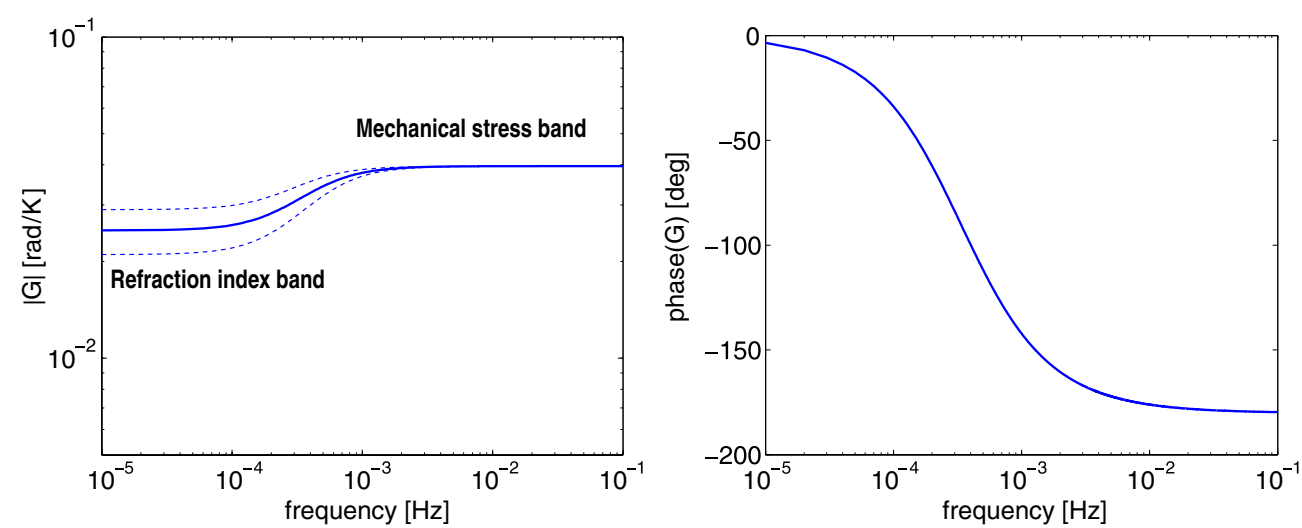

Figure 7. Bode diagram for the optical window $A R M A(2,1)$ transfer function estimate using the values from table 1. Left panel: its modulus; dashed lines show the $1 \sigma$ confidence region. Note that this confidence region has been extrapolated below $\sim 0.4 \mathrm{mHz}$, as actual experimental data were actually not available in that band. Right panel: phase of the transfer function.

We now try to produce some insight into the physical meaning of the just discussed analysis facts.

\subsection{Physics of the ARMA process}

Two different kinds of thermal effects have been identified as sources of changes in the optical path length of a light beam traversing a plane-parallel piece of glass:

(i) Temperature-dependent changes of the refractive index

(ii) Mechanical stress induced changes of the refractive index

We briefly describe below how these effects can be approximately evaluated.

4.1.1. Temperature-dependent changes of the refractive index. The first effect, which is found under stress-free conditions, is quantified by the formula [16]

$$
\left.\frac{\mathrm{d} \phi}{\mathrm{d} T}\right|_{\text {free }}=2 \pi \frac{L}{\lambda}\left[\frac{\mathrm{d} n}{\mathrm{~d} T}+(n-1) \alpha_{E}\right],
$$

where $\phi$ is the phase shift suffered by a beam of light traversing a glass slab of thickness $L$ and (nominal) index of refraction $n ; \lambda$ is the wavelength of the used light, and $\alpha_{E}$ is the linear thermal expansion factor of the glass, $\alpha_{E}=L^{-1} \mathrm{~d} L / \mathrm{d} T$.

The $\mathrm{d} \phi /\left.\mathrm{d} T\right|_{\text {free }}$ effect is most prominent at very low frequencies and DC. The reason is that it happens even if the temperature of the glass is homogeneous, and without mechanical stresses. It has been measured on naked glass samples in the laboratory, free of any pressure or tension, with the result that it is $25 \mathrm{mrad} \mathrm{K}^{-1}$ [17], a figure very well matching the one given by equation (20). One, however, needs to consider that the latter was obtained from data of a real window, i.e., including metal flange. This consequently means that the stress contribution $\mathrm{d} \phi / \mathrm{d} \sigma$ must be comparatively small at very low frequencies.

The same result is endorsed by further independent evidence. If data-sheet properties of the OHARA S-PHM52 glass used in the experiment are used to calculate the thermal-related path-length variations in the optical window glass due to changes in the refractive index, the result is that $\mathrm{d} \phi /\left.\mathrm{d} T\right|_{\text {free }}$ is $\sim 21 \mathrm{mrad} \mathrm{K}^{-1}$, again in good agreement with equation (20). 


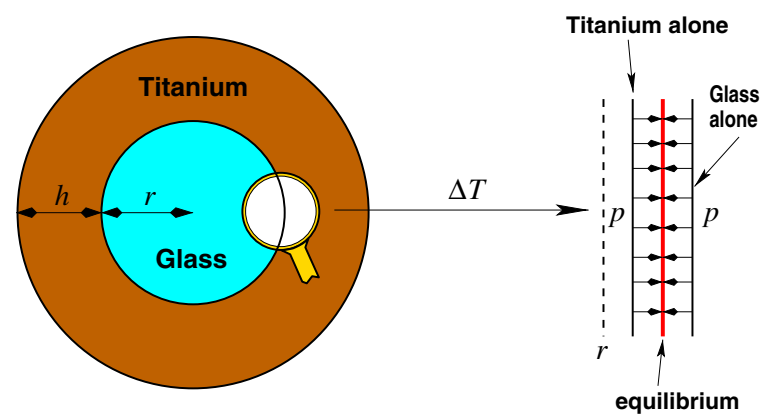

Figure 8. Schematics of the dilatation of the OW glass and the clamping titanium flange: the right part zooms in the profiles acquired by the interface (red-in the electronic version only) when the temperature of the block increases by $\Delta T$. Should either the glass or the titanium be allowed to expand freely, the boundaries would be placed as also represented. The dashed line is the interface position before heating.

4.1.2. Mechanical stress induced changes of the refractive index. This second effect is relevant to our experiment because the glass, clamped by titanium flanges to the ISH structure, is under stress due to differing thermal expansion coefficients in glass and metal. Mechanical stress also induces pathlength changes which are difficult to model. From the datasheet, the only parameter provided by the manufacturer which can be used to quantify these interactions is the photoelastic coefficient, $\beta$. However, it must be noted that $\beta$ does not describe the change in the refractive index due to stress, $\mathrm{d} n / \mathrm{d} \sigma$, but the appearance of birefringence due to stress, i.e., the change of the velocity of light along different axes of the material. Although not directly related, both parameters range in the same order of magnitude [16], and we shall thus use the photoelastic coefficient here for our order of magnitude estimate, described in the following.

Under this simplifying assumption, the photoelastic coefficient can be related to a pathlength variation by

$$
\Delta s_{\text {stress }}=\beta \sigma d
$$

where $\beta=10^{-5} \mathrm{~nm} \mathrm{~cm}^{-1} \mathrm{~Pa}^{-1}, d$ is the glass thickness ( $d=0.6 \mathrm{~cm}$ for the optical window) and $\sigma$ is the applied stress, having dimensions of pressure.

In this case, the stress on the glass is due to differential thermal dilatation of the titanium flange and the OW glass itself. The situation is illustrated graphically in figure 8 . Because the coefficient of thermal expansion of the glass is larger than that of the titanium flange embracing it, the latter expands less when submitted to the same temperature rise, and hence the glass is compressed radially along the rim. The opposite happens if the temperature decreases, i.e., the glass is in this case stretched outwards by the radial pull of the titanium. The contraction/expansion forces acting on glass and titanium reach an equilibrium state which determines the radii of the contracted/expanded pieces of titanium and glass. The equilibrium position thus happens when

$$
\left[\delta \rho_{T}+\delta \rho_{\sigma}\right]_{\mathrm{Ti}}=\left[\delta \rho_{T}-\delta \rho_{\sigma}\right]_{\text {Glass }},
$$

where $\delta \rho_{T}$ and $\delta \rho_{\sigma}$ refer to changes in radius caused by temperature changes and by stresses, respectively. The above formula holds even if temperature changes in titanium and glass are unequal. On the other hand, we are not considering in our description possible effects coming from the helicoflex ring between the titanium and the glass. As stated above, we are here trying to get an order of magnitude of the effect based on a simplified mechanical model, and interface effects are thus not included. 
The contributions appearing in equation (23) are given by [18]

$$
\delta \rho_{T}=\rho \alpha \Delta T \quad \text { and } \quad \delta \rho_{\sigma}=\frac{p \rho^{2}}{\ell E},
$$

where $\rho$ is the radius of the interface between the titanium and the glass, $\ell$ stands for the width of the body, $E$ is the Young's modulus, $\alpha$ is the thermal expansion coefficient and $p$ is the lateral pressure. Combining equations (23) and (24), and following the notation of figure 8 , we find the lateral pressure on the glass:

$$
p=\frac{\alpha_{\mathrm{Ti}} \Delta T_{\mathrm{Ti}}-\alpha_{\mathrm{Glass}} \Delta T_{\mathrm{Glass}}}{(r / h) E_{\mathrm{Ti}}^{-1}+E_{\mathrm{Glass}}^{-1}} .
$$

The strain on the glass lateral surface is given by $\sigma_{\mathrm{Glass}}=\mathrm{prd} /(\mathrm{rd})=p$, where $d$ is the thickness of the window glass (see [18]). Hence,

$$
\sigma_{\mathrm{Glass}}=\frac{\alpha_{\mathrm{Ti}} \Delta T_{\mathrm{Ti}}-\alpha_{\mathrm{Glass}} \Delta T_{\mathrm{Glass}}}{E_{\mathrm{Ti}}^{-1}+(h / r) E_{\mathrm{Glass}}^{-1}} .
$$

We can consider two different regimes here: the low-frequency (LF) regime and the high-frequency $(\mathrm{HF})$ regime. The first corresponds to long duration heat pulses applied on the titanium flange, actually long enough that the temperatures of the glass and the titanium are equal to each other, or $\Delta T_{\mathrm{Ti}}=\Delta T_{\mathrm{Glass}} \equiv \Delta T$. In this case,

$$
\sigma_{\mathrm{Glass}}=\frac{\alpha_{\mathrm{Ti}}-\alpha_{\mathrm{Glass}}}{E_{\mathrm{Ti}}^{-1}+(h / r) E_{\mathrm{Glass}}^{-1}} \Delta T, \quad \text { low frequency. }
$$

On the other hand, if short heat pulses are applied on the titanium then the glass does not have time to respond, and in this we can assume $\Delta T_{\mathrm{Glass}}=0$ and $\Delta T_{\mathrm{Ti}} \equiv \Delta T$. Thus,

$$
\sigma_{\mathrm{Glass}}=\frac{\alpha_{\mathrm{Ti}}}{E_{\mathrm{Ti}}^{-1}+(h / r) E_{\mathrm{Glass}}^{-1}} \Delta T, \quad \text { high frequency }
$$

We can use the above formulae in combination with (22) to obtain

$$
\left.\frac{\mathrm{d} \phi}{\mathrm{d} T}\right|_{\text {Stress }}= \begin{cases}\beta \frac{2 \pi d}{\lambda_{\text {laser }}} \frac{\alpha_{\mathrm{Ti}}-\alpha_{\mathrm{Glass}}}{E_{\mathrm{Ti}}^{-1}+(h / r) E_{\mathrm{Glass}}^{-1}}, & \text { low frequency } \\ \beta \frac{2 \pi d}{\lambda_{\text {laser }}} \frac{\alpha_{\mathrm{Ti}}}{E_{\mathrm{Ti}}^{-1}+(h / r) E_{\mathrm{Glass}}^{-1}}, & \text { high frequency. }\end{cases}
$$

It is recalled that $\Delta \phi=2 \pi \Delta s / \lambda_{\text {laser }}$, where $\lambda_{\text {laser }}$ is the laser wavelength. We put numbers here:

to obtain

$$
\begin{aligned}
& \beta=10^{-3} \times 10^{-9} \mathrm{~Pa}^{-1} \\
& d=6 \times 10^{-3} \mathrm{~m} \\
& \lambda_{\text {laser }}=1.064 \times 10^{-6} \mathrm{~m} \\
& \alpha_{\mathrm{Ti}}=8.6 \times 10^{-6} \mathrm{~K}^{-1} \\
& E_{\mathrm{Ti}}=11.6 \times 10^{10} \mathrm{~N} \mathrm{~m}^{-2} \\
& h=0.02 \mathrm{~m} \\
& \alpha_{\text {Glass }}=10 \times 10^{-6} \mathrm{~K}^{-1} \\
& E_{\text {Glass }}=7.15 \times 10^{10} \mathrm{~N} \mathrm{~m}^{-2} \\
& r=0.015 \mathrm{~m}
\end{aligned}
$$

$$
\left.\frac{\mathrm{d} \phi}{\mathrm{d} T}\right|_{\text {Stress }}= \begin{cases}2.5 \times 10^{-3} \mathrm{rad} \mathrm{K}^{-1}, & \text { low frequency } \\ 15 \times 10^{-3} \mathrm{rad} \mathrm{K}^{-1}, & \text { high frequency. }\end{cases}
$$


Table 2. $\mathrm{d} \phi / \mathrm{d} T$, units in $\operatorname{mrad} \mathrm{K}^{-1}$

\begin{tabular}{lll}
\hline & ARMA & Analytic \\
\hline LF range & $25 \pm 4$ & 23.5 \\
HF range & 40 & 36 \\
Nude glass & - & 21 \\
\hline
\end{tabular}

4.1.3. Discussion of the results. The total thermal effect is the sum of the above two effects, i.e., optical pathlength changes induced by pure thermal expansion and by mechanical stress. The former gives a value of $21 \times 10^{-3} \mathrm{rad} \mathrm{K}^{-1}$ throughout the frequency band, as extracted from datasheet values (see section 4.1.1). We can thus summarize the results as shown in table 2 .

The agreement between the results produced by our simplified model and the ARMA fit is quite good. Even though the model is not fully comprehensive of all the physical effects happening in the OW, it can be considered rather satisfactory from a purely empirical point of view, hence very useful for practical purposes. Work is currently in progress for a more thorough approach, and we shall report on new results in due course.

We conclude from this discussion that the low-pass component of the transfer function is almost exclusively related to the $\mathrm{d} \phi /\left.\mathrm{d} T\right|_{\text {free }}$ effect, while the stress effects only show up significantly in the higher frequency band. This makes sense, as stresses applied along the glass rim quickly propagate inwards throughout the glass piece.

Although the LTP spectrum is only above $1 \mathrm{mHz}$, an analysis at frequencies below this, down to $10^{-4} \mathrm{~Hz}$ and even further, must be considered of high interest, as the latter frequency band will be important for LISA. The experimental data reported in this paper can be improved to access the lower LISA band, since they typically consist in $1 \mathrm{~h}$ long runs. This is a strong suggestion for the LTP experiment plan.

\section{Noise projection}

One of the main scientific objectives of the diagnostics system in the LTP is to measure identified environmental disturbances [8], and to provide the data and analysis tools to estimate the contribution of those disturbances to the overall mission noise budget, equation (1). In practice this means the LTP data and diagnostics subsystem (DDS) must be able to provide suitable transfer functions to convert measured disturbance noise into test mass acceleration noise. This section is devoted to describing this procedure in the case of temperature fluctuation noise in the OW, and to show how it works in an on-ground laboratory experiment — to be extrapolated to a space-borne one.

We will use the results derived in the previous analysis to obtain an estimation for the thermal contribution to the interferometer performance. We shall naturally limit ourselves to the ARMA model, since it is the one making sense for real mission purposes, as already discussed.

The basic idea is that the OW transfer function, as determined from high SNR system response, also applies when there is only (weaker) noise in the window [10]. For this we shall use the one in equation (11), i.e.,

$$
G(z, \alpha, \beta, \delta)=\alpha \frac{1-z^{-1}}{1+\beta z^{-1}}+\frac{\delta}{1+\beta z^{-1}} .
$$

We now show which procedures must be applied to address the problem of finding the contribution of temperature fluctuations noise in the OW to the total OW noise. To this end we 

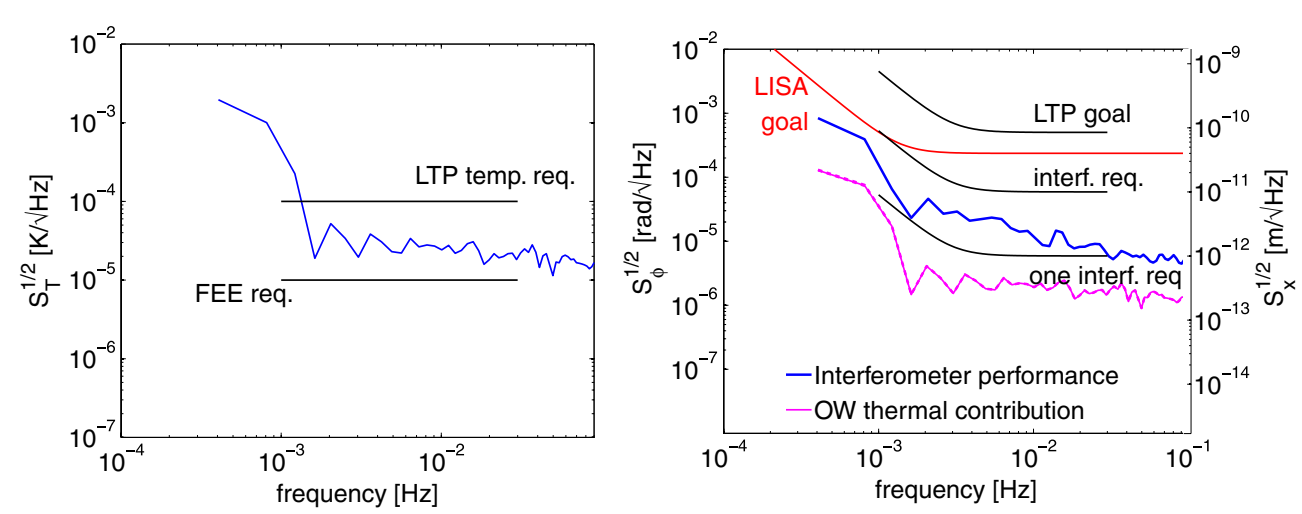

Figure 9. Temperature fluctuations spectrum (left) measured during the performance experiment compared to the LTP temperature requirement and the lower limit set by the front-end electronics sensitivity limit. Phase fluctuations (blue curve, right panel —in the electronic version only) during the same run are compared to the optical window thermal contribution (magenta curve, right panel-in the electronic version only), as derived with the ARMA transfer functions given by equations (32) and (17). The $1 \sigma$ confidence region is also included for the latter.

consider data of temperature and phase noise generated in a different experiment, and apply to it the methodology just sketched.

The laboratory setup and the experimental details can be found in [19]. In this experiment, the optical window is not a part of a testing optical bench (OB), but is glued in a lateral side of the LTP OB engineering model, instead. This way, a double beam pass across the window is forced: the laser light travels from the optical bench through the OW to a dummy mirror, faking a test mass; there, it is reflected, sent back again across the OW and out to the OB. Such a setup proved to be compliant with the interferometer noise budget, showing that the inclusion of the OW does not degrade the interferometer performance. Two temperature sensors in the titanium flange and one in the glass were left in place to measure temperature values during long-term runs.

No thermal disturbances were deliberately introduced, so the thermometers only read environmental temperature fluctuations. We use equation (17) to convert the temperature fluctuations spectral density, $S_{T}^{1 / 2}(\omega)$, into a phasemeter spectral density, $S_{\phi, T}^{1 / 2}(\omega)$. Thus,

$$
S_{\phi, T}^{1 / 2}(\omega)=2|\widetilde{G}(\omega, \alpha, \beta, \delta)| S_{T}^{1 / 2}(\omega)
$$

where the numerical factor 2 is required to account for the double passage of the laser beam through the window in this case. We assume both passages are completely correlated, given the extremely small time scale of their occurrence compared to thermal reaction times. Spectral densities are therefore linearly added.

Results obtained in a typical run with that setup are plotted in figure 9 using a MATLAB package being developed ex professo for the LTP data analysis [20]. The left panel shows temperature fluctuations measured in the titanium flange. As can be seen, these reach the front end electronics (FEE) floor noise in the higher frequency region of the measuring bandwidth, while keeping slightly above the LTP maximum temperature fluctuations requirements limit in the lower frequencies, around $1 \mathrm{mHz}$ [21]. This is, in fact, a worst-case condition, since the temperature power spectrum decreases as frequency increases, and thus if the LTP temperature requirement is reached at a lower frequency range then the rest of the spectrum will naturally follow a descending curve like the one shown in figure 9. 
The phasemeter fluctuations spectrum is however below the required noise level, as we can see in the blue curve of the right panel (in colour in the electronic version only). The temperature fluctuations spectral data in the left panel are then submitted to the algorithm, equation (32), and the result is the magenta curve displayed in the right panel (in colour in the electronic version only).

The low coupling to thermal disturbances implied by $\widetilde{G}(\omega, \alpha, \beta, \delta)$ causes the thermal contribution to only represent $5 \%$ of the phasemeter noise at $1 \mathrm{mHz}$, and about $0.5 \%$ of the LTP goal. We thus feel reassured that there is still considerable margin here.

\section{Continuous time models}

On account of the empirical results reported in section 3, and of the remarkable accuracy with which both DLR and ARMA models fit the experimental data - notwithstanding their completely different nature-we now try to shed some light on the kind of processes which take place in the system.

For this, we attempt to picture the $\operatorname{ARMA}(2,1)$ model relating the phase readout of the interferometer $\phi[n]$ and the temperature at the titanium flange $T_{\mathrm{Ti}}[n]$ as the digital implementation of some analogue physical process. The starting point is of course the digital algorithm, equation (7), which in this case is given by

$$
G(q, \alpha, \beta, \delta)=\alpha \frac{1-q^{-1}}{1+\beta q^{-1}}+\frac{\delta}{1+\beta q^{-1}},
$$

where $q$ is the shift operator of equation (8). The recursive form of the process thus defined is therefore

$$
\phi[n]+\beta \phi[n-1]=\alpha\left\{T_{\mathrm{Ti}}[n]-T_{\mathrm{Ti}}[n-1]\right\}+\delta T_{\mathrm{Ti}}[n]
$$

and can be regarded as the digital implementation of a first-order continuous time filter, governed by a first-order differential equation:

$$
\dot{\phi}(t)+\tau^{-1} \phi(t)=A \dot{T}_{\mathrm{Ti}}(t)+B T_{\mathrm{Ti}}(t), \quad\left(. \equiv \frac{\mathrm{d}}{\mathrm{d} t}\right),
$$

where $\tau$ is the characteristic time constant of the analogue filter, and $A$ and $B$ are scale factors, respectively, weighing the contributions of the temperature's time variation rate and the temperature itself to the phase-shift effect. We have assumed the $T_{\mathrm{Ti}}(t)$ dependence in the rhs of (35) in line with the fit result expressed by the rhs of (34).

If the time constant $\tau$ is much larger than the sampling time $\Delta t$ implicit in equation (34) then we can approximate time derivatives by

$$
\dot{\phi}(t) \simeq \frac{\phi(t)-\phi(t-\Delta t)}{\Delta t}
$$

and, mutatis mutandi, the same for $T_{\mathrm{Ti}}(t)$. Taking $t=n \Delta t$ for the timing of the $n$th sample, and using the natural notation $\phi[n] \equiv \phi(n \Delta t)$, equation (35) is approximated by

$$
\begin{gathered}
\phi[n]-\left(1+\frac{\Delta t}{\tau}\right)^{-1} \phi[n-1]=A\left(1+\frac{\Delta t}{\tau}\right)^{-1}\left\{T_{\mathrm{Ti}}[n]-T_{\mathrm{Ti}}[n-1]\right\} \\
+B \Delta t\left(1+\frac{\Delta t}{\tau}\right)^{-1} T_{\mathrm{Ti}}[n] .
\end{gathered}
$$

This can be readily compared to equation (34) to obtain

$$
\beta=-\left(1+\frac{\Delta t}{\tau}\right)^{-1}, \quad \alpha=A\left(1+\frac{\Delta t}{\tau}\right)^{-1}, \quad \delta=B \Delta t\left(1+\frac{\Delta t}{\tau}\right)^{-1} \text {. }
$$


$\beta$ is seen to have a value very close to -1 (table 1$)$, or $\beta=-(1-\eta)$ with $\eta<10^{-2}$ comfortably in all cases. Hence $\tau \simeq \Delta t / \eta$, i.e., $\tau \gg \Delta t$, which a posteriori justifies the approximation leading to equation (37).

The formal solution to equation (35) can be easily written. After initial transients die out, the phase is given by

$$
\phi(t)=A T_{\mathrm{Ti}}(t)+(B-A) \tau^{-1} \int_{0}^{t} \mathrm{e}^{-\left(t-t^{\prime}\right) / \tau} T_{\mathrm{Ti}}\left(t^{\prime}\right) \mathrm{d} t^{\prime}
$$

The meaning of this filter equation is better understood if we recast it in the frequency domain:

$$
\widetilde{\phi}(\omega)=\left[A \frac{\mathrm{i} \omega \tau}{1+\mathrm{i} \omega \tau}+B \frac{\tau}{1+\mathrm{i} \omega \tau}\right] \widetilde{T}_{\mathrm{Ti}}(\omega)
$$

This equation shows again that the analogue process is also the superposition of two contributions: a high-pass filter proportional to $A$ and a low-pass contribution proportional to $B$. The first arises in equation (34) due to the titanium temperature derivative, while the second appears related to the term proportional to the titanium absolute temperature. This split dependence of the OW response to temperature pulses points to two different physical thermal processes affecting the glass, as already discussed in section 4.1.

We can now make use of equations (38) to identify the coefficients $A$ and $B$ in terms of the fit parameter values of table 1 . Taking $\Delta t / \tau \ll 1$, we find that $A \simeq \alpha$, and $B \simeq \delta / \Delta t$. In addition, we can take advantage of the relationship $\alpha \simeq p_{1}$ between the auto-regressive and the DLR model parameters to obtain an expression relating both models. Accordingly, equation (39) can be rewritten as

$$
\phi(t) \simeq p_{1} T_{\mathrm{Ti}}(t)+\left(\delta / \Delta t-p_{1}\right) \tau^{-1} \int_{0}^{t} \mathrm{e}^{-\left(t-t^{\prime}\right) / \tau} T_{\mathrm{Ti}}\left(t^{\prime}\right) \mathrm{d} t^{\prime} .
$$

If we go back to the DLR fit formula, equation (2), the following expression ensues:

$$
T_{\text {Glass }}(t) \simeq-\frac{p_{1}}{p_{2}} \tau^{-1} \int_{0}^{t} \mathrm{e}^{-\left(t-t^{\prime}\right) / \tau} T_{\mathrm{Ti}}\left(t^{\prime}\right) \mathrm{d} t^{\prime},
$$

after the term $\delta / \Delta t$ has been safely neglected in front of $p_{1}$. We thus see that temperatures in the titanium flange and in the OW glass are related by a low pass with a time constant, $\tau$, of a few hundred seconds - note that $p_{1}$ and $p_{2}$ have different signs, table 1 .

It must be recalled that this relationship emerges out of the good quality of the fits by both DLR and ARMA(2,1) models, and is key to understanding why only the titanium gauge is required to make a good prediction of the OW response to temperature variations, as will be required in flight. The physical reason for the observed relationship between temperatures is to be sought in the properties of the interface between the titanium and the glass in the OW.

\section{Conclusions}

While the optical window is a crucial element in the LTP optical metrology system, it thankfully appears that it is quite stable to temperature fluctuation noise-so far as the latter is compliant with mission environmental requirements. The present paper contains a rather thorough analysis of such behaviour, based on experimental data gathered through different runs of on-ground laboratory measurements.

Our main purpose was to prepare for thermal diagnostics analysis tools in flight, and to gain as much understanding of the underlying physical processes as possible. This means we need to know how noisy data retrieved by thermometers can be converted into phasemeter fluctuations, thereby quantifying the contribution of temperature random variations to the 
total mission noise budget-which is the ultimate objective of LPF in preparation for LISA. Our most relevant finding is the discovery that temperature readings in the titanium flange embracing the OW plane-parallel plate relate to phase values through an $\operatorname{ARMA}(2,1)$ transfer function. Although this is the result of numerical analysis, hence lends itself to parameter estimation variances, it appears to be considerably robust.

The analysis has shown that the $A R M A(2,1)$ process naturally splits up as the sum of a high-pass and a low-pass process, each of them with significantly different relative weights which result in the high-pass dominating above $\sim 1 \mathrm{mHz}$, while the low pass takes over in the lower LISA frequency band, i.e., at $0.1 \mathrm{mHz}$ and below. A major achievement of the analysis has been the identification of the physical processes responsible for this behaviour: mechanical stresses - induced by differential thermal expansion of metal and glass - are associated with the high-pass term, while $\mathrm{d} \phi /\left.\mathrm{d} T\right|_{\text {free }}$ effects account for the low pass.

We consider the analysis presented here as rather complete in some of its essential traits. But there are still open issues which call for further study. For example, heater generation of test signals must be monitored by temperature sensors close to the activated heaters-due to lag effects in remoter spots-for the procedures described herein to be fully operative. This raises some caveats regarding full applicability of the noise projection algorithms, as the sources of heat dominating a given temperature reading may not be clear in LTP science operation mode.

A more global tool for full LTP thermal diagnostics, which takes into account the specific features of each individual part of the system, must be assembled. Research on this is currently underway which will be reported in due course.

\section{Acknowledgments}

Support for this work came from Project ESP2004-01647 of Plan Nacional del Espacio of the Spanish Ministry of Education and Science (MEC). MN acknowledges a grant from Generalitat de Catalunya and JS a grant from MEC.

\section{References}

[1] Bender P et al 2000 Laser Interferometer Space Antenna: a cornerstone mission for the observation of gravitational waves ESA Report No ESA-SCI(2000)11

[2] Anza S et al 2005 The LTP experiment on the LISA Pathfinder mission Class. Quantum Grav. 22 S125-38

[3] The LPF Science Team 2006 LISA PathFinder: Einstein's Geodesic Explorer ESA Report No ESA-SCI(2007)1

[4] The Project Engineering Team 2005 Study on implementation of inertial sensor vacuum achievement by in-orbit venting Report No S2-ASD-RP-3016

[5] Carbone L, Cavalleri A, Dolesi R, Hoyle C D, Hueller M, Vitale S and Weber W J 2003 Achieving geodetic motion for LISA test masses: ground testing results Phys. Rev. Lett. 91 151101-1

[6] Dolesi R et al 2003 Gravitational sensor for LISA and its technology demonstration mission Class. Quantum Grav. 20 S99-108

[7] Heinzel G et al 2004 The LTP interferometer and phasemeter Class. Quantum Grav. 21 S581-7

[8] Vitale S 2005 Science Requirements and Top-level Architecture Definition for the Lisa Technology Package $(L T P)$ on Board LISA Pathfinder (SMART-2) LPF report no. LTPA-UTN-ScRD-Iss003-Rev1

[9] The LISA International Science Team 2006 ESA-NASA Report No LISA-ScRD-004

[10] Lobo A 2005 DDS science requirements document LPF Report No S2-IEEC-RS-3002

[11] Lobo A et al 2005 LTP thermal diagnostics Class. Quantum Grav. 22 S171-6

[12] Ramos-Castro J et al 2005 DDS thermal diagnostic prototype design Tech. Rep. S2-UPC-DDD-3001

[13] Sanjuan J et al Thermal diagnostic front-end electronics for LISA Pathfinder, in preparation

[14] Kay S M 1988 Modern Spectral Estimation (Englewood Cliffs, NJ: Prentice-Hall)

[15] Ljung L 1999 System Identification: Theory for the User (Englewood Cliffs, NJ: Prentice-Hall)

[16] García Marín A F 2007 PhD Memoir Albert Einstein Institute, Hannover 
[17] García Marín A F et al 2006 On-orbit alignment and diagnostics for the LISA technology package Class. Quantum Grav. 23 S133-40

[18] Timoshenko S 1956 Strength of Materials (Princeton, NJ: Van Nostrand-Reinhold)

[19] García Marín A F et al 2006 Interferometric characterization of the optical window for LISA and LISA Pathfinder Proc. LISA Symp. No 6 ed S Merkowitz and J C Livas AIP Conf. Proc. 873 344-8

[20] http://www.lisa.uni-hannover.de/ltpda

[21] Lobo A, Nofrarias M, Ramos-Castro J and Sanjuan J 2006 On-ground tests of the LISA PathFinder thermal diagnostics system Class. Quantum Grav. 23 5177-93 